\title{
Production and $\beta$ half-lives of heavy neutron-rich nuclei approaching the r-process path at $\mathrm{N}=126$
}

\author{
J. Benlliure*; H. Alvarez-Pol, T. Kurtukian-Nieto; A.I. Morales, E. Casarejos, D. \\ Cortina-Gil, J. Pereira \\ Universidad de Santiago de Compostela, 15782 Santiago de Compostela, Spain
}

\author{
F. Becker, I. Borzov, T. Enqvist, D. Henzlova, K. Langanke, G. Martínez-Pinedo, \\ K.-H. Schmidt, O. Yordanov \\ GSI Helmholtzzentrum für Schwerionenforschung GmbH, Planckstr. 1, D-64291 Darmstadt, \\ Germany
}

\section{B. Blank, J. Giovinazzo, B. Jurado}

Université Bordeaux I, CNRS/IN2P3, CENBG, BP 120, F-33175 Gradignan, France

\section{Audouin, P. Napolitani}

IPN, IN2P3-CNRS, Université Paris-Sud 11, UMR 8608, F-91406 Orsay, France

\author{
B. Fernández, F. Rejmund \\ GANIL CEA/DSM-CNRS/IN2P3, BP 55027, F-14076 Caen, France
}

\section{and the RISING collaboration}

\begin{abstract}
Fragmentation reactions of relativistic ${ }^{238} \mathrm{U}$ and ${ }^{208} \mathrm{~Pb}$ projectiles have been used to investigate the production of heavy neutron-rich nuclei approaching the r-process waiting point at $\mathrm{A} \approx 195$. The relativistic energies, together with the use of a high resolving-power magnetic spectrometer were key conditions for the unambiguous identification of nuclei in the region of interest. Using this technique we were able to identify 73 new heavy neutron-rich nuclei expanding considerably the north-west frontier of the chart of nuclide. Moreover, we were able to determine the half lives of 13 of those nuclide. The measured values are significantly shorter than the predictions used for r-process model calculations. The confirmation of these results for r-process nuclei at $\mathrm{A} \approx 195$ would indicate that the r-process at this point is faster than expected, leading to a larger production of the heaviest nuclei.
\end{abstract}

11th Symposium on Nuclei in the Cosmos

19-23 July 2010

Heidelberg, Germany. 


\section{Introduction}

One of the most challenging contributions of nuclear physics to the understanding of the stellar nucleosynthesis r-process is probably the production and determination of ground properties of r-process nuclei at the $\mathrm{N}=126$ shell closure. During the last years, existing radioactive beam facilities helped us to progress a lot in the production and characterization of medium-mass r-process nuclei $[1,2,3]$. However, the region of heavy neutron-rich nuclei remained almost unexplored despite its interest. This region plays an important role in fundamental nuclear structure investigations, because of the presence of the neutron shell $\mathrm{N}=126$, but it is also extremely relevant for the characterization of the $r$-process at the waiting point around $A \approx 195$. Indeed, the r-process matter flow towards the heaviest elements is to a large extend determined by the half lives of the r-process nuclei at the $\mathrm{N}=126$ waiting point. Unfortunately, present model descriptions of the beta half lives of heavy neutron-rich nuclei are rather discordant $[5,6]$. It was then the purpose of this work to investigate the production of heavy neutron-rich nuclei but also to measure the $\beta$ half lives of some of them in order to benchmark the existing theoretical predictions.

\section{Production of heavy neutron-rich nuclei}

In order to produce heavy neutron-rich nuclei we proposed to use fragmentation reactions of relativistic projectiles. Although fragmentation reactions produce mostly neutron-deficient nuclei, the large fluctuations in the nature of the abraded nucleons (protons or neutrons) and in the excitation energy gained in the collision make it possible to populate cold fragmentation reaction channels. These reaction channels are characterized by the abrasion of mostly protons while the excitation energy gained in the process is rather low, limiting then the evaporation of neutrons. It was shown some years ago [7] that these reaction channels produce the most neutron-rich nuclei in fragmentation reactions, in particular proton-removal channels where only protons are abraded and no neutrons are evaporated.

In this particular case we used beams of ${ }^{238} \mathrm{U}$ and ${ }^{208} \mathrm{~Pb}$ projectiles accelerated at $1 \mathrm{~A} \mathrm{GeV}$ at the SIS18 synchrotron in GSI. Pulsed beams with a typical bunch length of 3 seconds a repetition rate of 10 seconds and a typical intensity of $\approx 10^{8}$ ions/s where extracted from the accelerator and directed to a $2 \mathrm{~g} / \mathrm{cm}^{2}$ thickness beryllium target. Projectile fragments, flying forward because of the kinematical conditions, were analyzed with the magnetic spectrometer FRagment Separator (FRS) [8]. This is a zero-degree magnetic spectrometer with two symmetric sections in order to preserve the achromatism of the system. A profiled energy degrader placed at the intermediate image plane was fundamental for the separation of the transmitted nuclei [9] and the identification of atomic charge states. The nuclei traversing the spectrometer were identified by determining their magnetic rigidity, velocity and atomic number. A description of the identification method for these nuclei can be found in $[10,11]$.

In figure 1 we represent on top of chart of nuclide all nuclei produced in this work. As can be seen we were able to produce 73 new neutron-rich isotopes of elements between Ytterbium and

\footnotetext{
* Speaker.

${ }^{\dagger}$ E-mail: j.benlliure@usc.es

†Present address: Centre d'Etudes Nuclèaires Bordeaux, Gradignan, France
} 


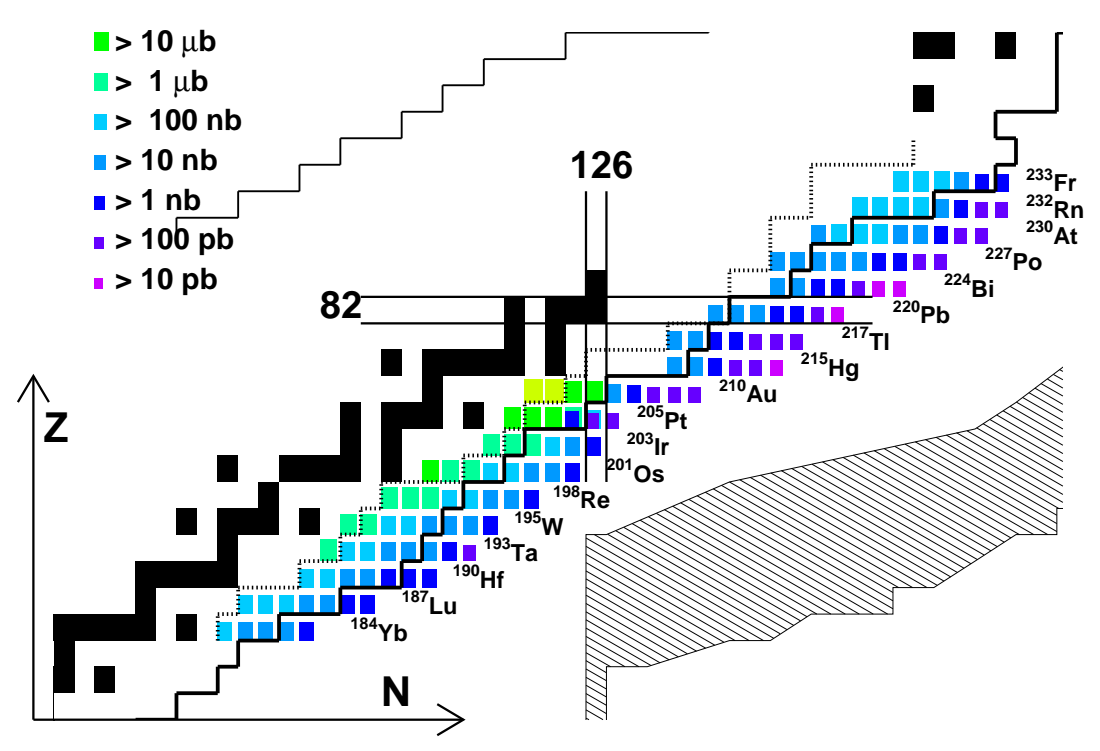

Figure 1: Nuclei produced in this work on top of a chart of nuclide, the color scale represents intervals of production cross section. Black squares correspond to stable nuclei. The dotted line represents the limit of known nuclei as in 1958 and the thick-solid line the limit of known nuclei before these experiments. The hatched area corresponds to an estimation of the path followed by the stellar nucleosynthesis r-process.

Francium. Some of these results were previously reported in $[12,13]$. The present results constitute a major step towards the production of nuclei at the $\mathrm{A} \approx 195$ r-process waiting point. Indeed in Fig. 1 the dotted line indicates the limits of the chart of nuclide as in 1958. Therefore, the present experiment allowed us to produce in few days of beam time as many new nuclei in this region of the chart of nuclide as in the previous fifty years.

In this work we could also determine the production cross sections of these nuclei normalizing the production yields to the integrated beam current and the number of target nuclei. The beam current was continuously measured during the experiment using a secondary-electron monitor placed before the reaction target. This monitor was carefully calibrated during the experiment using a plastic scintillator [10]. The measured yields were also corrected by the losses inherent to the experimental technique used in this work. The main corrections were due to the limited momentum acceptance of the spectrometer, in particular, for those nuclei with magnetic rigidities far from the central value defined by the FRS tune, the fraction of non fully stripped nuclei, and secondary reactions in all layers of matter placed along the spectrometer. Smaller corrections were due to the acquisition dead time and detectors efficiency. These cross sections were used to benchmark model calculations predicting the production of heavy neutron-rich nuclei in future radioactive beam facilities. These calculations clearly show that new radioactive beam facilities presently under design or construction will allow us to produce nuclei at the $\mathrm{A} \approx 195$ r-process waiting point. 


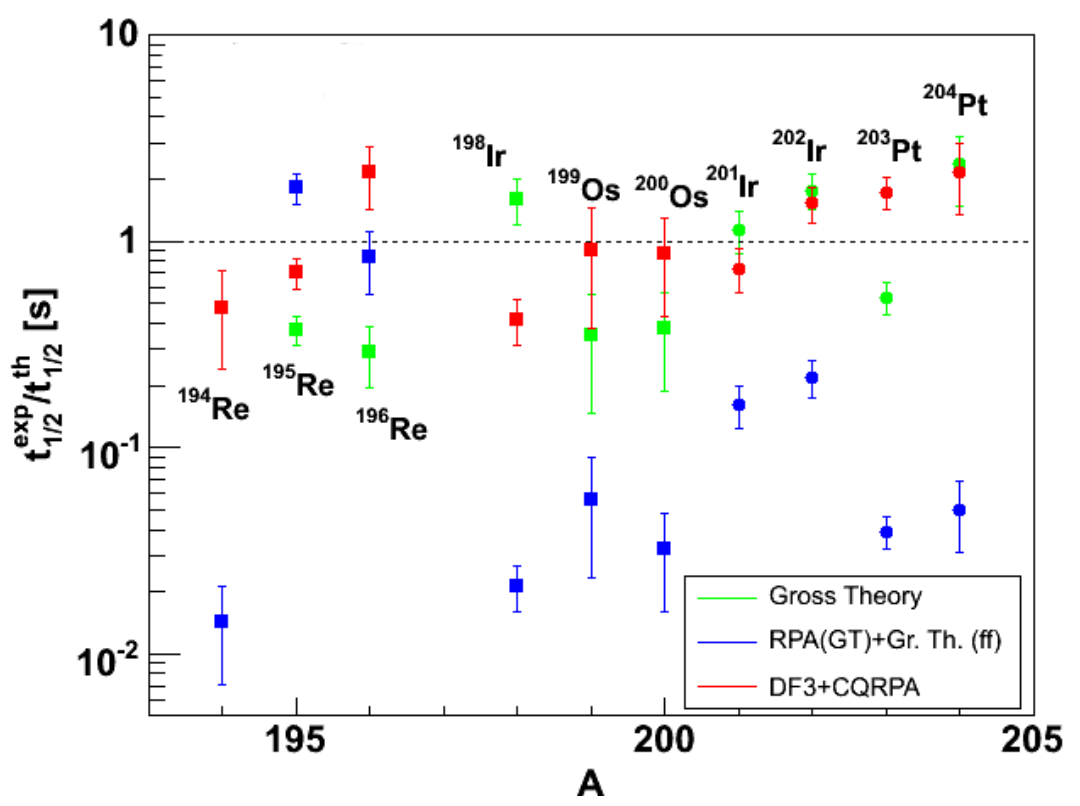

Figure 2: Half lives of heavy neutron-rich nuclei measured in this work normalized to different theoretical predictions, gross theory (green symbols), finite-range droplet model [5] (blue symbols) and energy-density functionals (red symbols) [6]. The measured half-lives for ${ }^{198} \operatorname{Ir}(8 \pm 2 \mathrm{~s}),{ }^{199} \operatorname{Ir}(6 \pm 4 \mathrm{~s})$ and ${ }^{204} \mathrm{Au}(37 \pm 1 \mathrm{~s})$ are not shown for the clarity of the figure.

\section{Half-lives of heavy neutron-rich nuclei}

Some of the nuclide produced in this experiment were slowed down and implanted into an array of three double-sided silicon strip detectors with a thickness of $1 \mathrm{~mm}$ [14]. This array provided the position and arriving time of the nuclide and the position and emission time of the subsequent $\beta$ particles. From the position and time correlations we were able to determine the $\beta$ half lives of the nuclide of interest. Because of the pulsed nature of the beam and the relatively long half lives of the nuclide we were investigating, we could not use standard techniques for the determination of the half lives. In this case, we proposed to use backward-time correlations for evaluating our background and numerical fitting functions based on Monte Carlo simulations. A detailed description of this method can be found in Ref. [15].

In figure 2 we reported the measured half lives normalized to the predictions obtained with three model calculations, the gross theory (green symbols), calculations based in the finite-range drop model by Moller et al.[5] (blue symbols) and calculations based in the energy-density functionals by Borzov [6] (red symbols). As can be seen, the Gross theory and Borzov's calculations provide a rather good description of the data. However, the calculations based on the finite-range droplet model of Moller et al. overestimates the half lives by almost two orders of magnitude for most of the nuclide investigated in this work [16].

Although we did not measured nuclides directly at the r-process, calculations for r-process Osmium isotopes indicate a similar difference between the model predictions [16]. The confirmation of the tendency we observed in this work as a general trend for r-process nuclei, would indicate 
that finite-range droplet model calculations would underestimate the speed of the r-process at the waiting point $A \approx 195$. Since these calculations are widely used in r-process simulations, these results would indicate that the r-process matter flow towards the heaviest elements would be faster than presently thought.

\section{Summary and conclusions}

In this work we have demonstrated that it is possible to produce heavy neutron-rich nuclei at the r-process waiting point $\mathrm{A} \approx 195$ using fragmentation reactions of heavy stable projectiles such us ${ }^{238} \mathrm{U}$ and ${ }^{208} \mathrm{~Pb}$ at relativistic energies. Indeed, in and experiment performed at GSI with modest primary beam intensities we were able to produce 73 new isotopes of elements between Ytterbium and Francium. Moreover, we could determine the $\beta$ half lives of thirteen of these nuclei. The measured half lives are rather well described with calculations based on the energy-density functionals while those calculations based on the finite-range droplet model largely overestimate the measured values. The confirmation of this overestimation of the $\beta$ half lives for nuclei at the $\mathrm{A} \approx 195 \mathrm{r}$-process waiting point would indicate that the matter flow at this waiting point is faster than predicted by present model calculations based on the finite-range droplet model. Therefore, the production of the heaviest r-process nuclei would also be larger than presently expected.

\section{References}

[1] J. Benlliure et al., Phys. Rev. C 78, 054605 (2008)

[2] K. Helariutta et al., Eur. Phys. J. A 17,181 (2003)

[3] T. Ohnishi et al., J. Phys. Soc. Jpn. 79, 073201 (2010)

[4] A. Jungclaus et al., Phys. Rev. Lett. 99, 132501 (2007)

[5] P. Moller et al., Phys. Rev. C 67, 055802 (2003)

[6] I. Borzov, Phys. Rev. C 67, 025802 (2003)

[7] J. Benlliure et al., Nucl. Phys. A 660, 87 (1999)

[8] H. Geissel et al., Nucl. Instr. and Methods B 70, 286 (1992)

[9] K.-H. Schmidt et al., Nucl. Instr. and Methods A 260, 287 (1987)

[10] E. Casarejos et al., Phys. Rev. C 74, 044612 (2006)

[11] J. Taïeb et al., Nucl. Phys. A 724, 413 (2003)

[12] H. Alvarez-Pol et al., Eur. Phys. J. A 42, 485 (2009)

[13] H. Alvarez-Pol et al., Phys. Rev. C 82, 041602 (2010)

[14] R. Kumar et al., Nucl. Instrum. and Methods A 598, 754 (2009)

[15] T. Kurtukian-Nieto et al., Nucl. Instrum. and Meth. A 589, 472 (2008)

[16] T. Kurtukian-Nieto et al., Phys. Lett. B submitted (arXiv:0711.0101v1) 